\title{
e-Migrinter
}

15 | 2017

Migrinter a trente ans : analyses et portraits

\section{Présentation du « Réseau Migrations »}

Josepha Milazzo, Jordan Pinel et Ewa Tartakowsky

\section{OpenEdition}

Journals

Édition électronique

URL : https://journals.openedition.org/e-migrinter/831

DOI : 10.4000/e-migrinter.831

ISSN : 1961-9685

Éditeur

UMR 7301 - Migrinter

Référence électronique

Josepha Milazzo, Jordan Pinel et Ewa Tartakowsky, «Présentation du « Réseau Migrations » », eMigrinter [En ligne], 15 | 2017, mis en ligne le, consulté le 20 mai 2021. URL : http://

journals.openedition.org/e-migrinter/831; DOI : https://doi.org/10.4000/e-migrinter.831

Ce document a été généré automatiquement le 20 mai 2021.

Tous droits réservés 
Présentation du « Réseau Migrations »

Josepha Milazzo, Jordan Pinel et Ewa Tartakowsky 
1 Le Réseau Migrations est un réseau international indépendant pluri et transdisciplinaire en sciences humaines et sociales. Il réunit de jeunes chercheurseuses $^{1}$ en cours de professionnalisation et travaillant tout ou partie sur les questions de migrations internationales. Fort de 170 membres environ, le Réseau associe également à sa liste de diffusion d'informations des chercheurs confirmés, des membres associatifs et de la société civile, qui ont un intérêt pour les activités du Réseau et les migrations internationales.

2 Le Réseau a été créé à l'initiative pictavienne de doctorants du laboratoire de recherche Migrinter lors d'un premier Séminaire annuel inaugural tenu en juin 2014 à l'Université de Poitiers. Le fonctionnement du Réseau repose depuis sur une charte ${ }^{2}$ qui définit ses activités, ses objectifs ainsi que son organisation constituée de trois pôles (la coordination, l'événementiel et la valorisation). Il dispose d'une liste de diffusion électronique, d'une plateforme numérique sur hypothèses.org ${ }^{3}$ ainsi que d'une page Facebook ${ }^{4}$ via lesquelles sont partagées et promues diverses informations, de même que les activités scientifiques entreprises par le Réseau et ses membres. Faisant suite aux événements du Réseau, plusieurs projets de publications ${ }^{5}$ dans des revues et ouvrages scientifiques ont vu le jour avec pour objectif de valoriser les travaux de ses membres.

3 Travaillant à la coopération, et avec l'appui financier et scientifique de divers partenaires institutionnels, associatifs et non-académiques nationaux et internationaux, le Réseau se donne pour mission de solidifier les liens entre jeunes chercheurs, en leur facilitant des lieux et moments de réflexion, de soutien et d'échange de savoir-(faire), notamment lors de rendez-vous scientifiques réguliers ou ponctuels 6 .

Pour la coordination du Réseau Migrations

reseau.migrations@gmail.com

\section{NOTES}

1. Parmi lesquels se trouvent des doctorant-e-s, des étudiant-e-s en master, des post-doctorant-e$\mathrm{s}$, et des chercheurs/ses non-titulaires rattaché-e-s à des institutions de l'enseignement et de la recherche.

2. http://reseaumig.hypotheses.org/charte

3. https://reseaumig.hypotheses.org/

4. https://www.facebook.com/reseaumig/

5. Voir notamment le numéro 14 de la revue e-Migrinter, qui constitue la première publication rassemblant de jeunes chercheurs, doctorants et docteurs, membres du Réseau Migrations: http://e-migrinter.revues.org/676. 
6. Des ateliers scientifiques informels trimestriels parfois jumelés avec des journées locales d'étude, un Séminaire annuel, une Université thématique biannuelle et des événements scientifiques indépendants organisés par ses membres et auxquels peut s'associer le Réseau.

\section{AUTEURS}

JOSEPHA MILAZZO

Doctorante en géographie, Aix-Marseille Université, TELEMMe / Universitat Autònoma de Barcelona, Departamento de Geografía

josepha.milazzo@yahoo.fr

JORDAN PINEL

Doctorant en géographie, Migrinter - UMR 7301 CNRS/Université de Poitiers

jordan.pinel@gmail.com

\section{EWA TARTAKOWSKY}

Docteure en sociologie, Centre Max Weber etartakowsky@yahoo.fr 